\title{
CURSO DIÁRIO E SAZONAL DAS TROCAS GASOSAS E DO POTENCIAL HÍDRICO FOLIAR EM ACEROLEIRAS ${ }^{1}$
}

\author{
REJANE JUREMA MANSUR CUSTÓDIO NOGUEIRA², \\ JOSÉ ANTÔNIO PROENÇA VIEIRA DE MORAES ${ }^{3}$ e HÉLIOALMEIDA BURITY4
}

\begin{abstract}
RESUMO - Este trabalho objetivou avaliar o curso diário e sazonal das trocas gasosas, da temperatura foliar e do potencial hídrico da acerola (Malpighia emarginata D.C.), no campo. O experimento realizou-se no município de Paudalho, PE. Os valores da transpiração e do potencial da água foram, de modo geral, mais elevados no início da manhã e no final da tarde; os da resistência difusiva e temperatura foliar foram menores no início da manhã e no final da tarde. Houve uma limitação das trocas gasosas com o ambiente, em decorrência da redução da transpiração nas horas mais quentes do dia, sendo mais acentuada na estação seca e na matriz UFRPE 7. Os valores mínimos do potencial ocorreram na época seca, variando de -3,4 MPa (UFRPE 7) a -4,3 MPa (UFRPE 8), enquanto os valores máximos da resistência

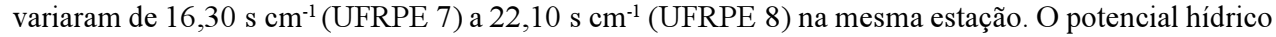
e a resistência difusiva mostraram forte correlação com o déficit de pressão de vapor. A maior capacidade fotossintética foi verificada em folhas maduras da matriz UFRPE 8. Os mecanismos fisiológicos apresentados pelas plantas demonstram que elas podem resistir a períodos de estresse hídrico quando estes se manifestam. A matriz UFRPE 8 é mais adaptada a períodos de estiagem do que a UFRPE 7.
\end{abstract}

Termos para indexação: Malpighia emarginata, relação planta-água, folhas, fotossíntese, transpiração, resistência à temperatura, estresse hídrico.

\section{DAILY AND SEASONAL COURSE OF GAS EXCHANGE AND LEAF WATER POTENTIAL IN ACEROLA PLANTS}

\begin{abstract}
The daily and seasonal course of the gas exchanges, leaf temperature and water potential of Barbados cherry (Malpighia emarginata D.C.) were evaluated under field conditions. The experiment was carried out in Paudalho, Pernambuco State, Brazil. Measurements of photosynthetic capacity in the wet season were also made. Changes in daily and seasonal behavior of gas exchange, water potential, and leaf temperature were observed. The transpiration and water potential measurements were higher at the beginning of the morning and at the end of the afternoon, while those for diffusive resistance and leaf temperature were lower at these same periods of the day. There was a reduction of gas exchange with the environment as a result of the lower transpiration during the hotter hours of the day, being this tendency strongest in the dry season and in the UFRPE 7 accession. The lowest values for leaf water potential were registered in the dry season, varying from $-3.37 \mathrm{MPa}$ (UFRPE 7) to $-4.32 \mathrm{MPa}$ (UFRPE 8), while the maximum resistance values varied from $16.30 \mathrm{~cm} \mathrm{~s}^{-1}$ (UFRPE 7) to $22.10 \mathrm{~cm} \mathrm{~s}^{-1}$ (UFRPE 8) during this same season. The water potential and the diffusive resistance showed a strong correlation to the vapour pressure deficit. The greatest photosynthetic capacity was registered for mature leaves in the UFRPE 8 plant. The physiological expressions displayed showed that the plants are resistant to drought and these expressions appear to help the plants resist to periods of water stress when they appear. The UFRPE 8 accession is more adapted to dry period than UFRPE 7. Index terms: Malpighia emarginata, plant water relations, leaves, photosynthesis, transpiration, temperature resistance, water stress.
\end{abstract}

\footnotetext{
${ }^{1}$ Aceito para publicação em 24 de novembro de 1999.

Extraído da tese de doutorado da primeira autora apresentada à Universidade Federal de São Carlos (UFSCar), SP.

${ }^{2}$ Biól, Dra., Dep. de Biologia, Universidade Federal Rural de Pernambuco (UFRPE), Rua Dom Manoel de Medeiros, s/no, Dois Irmãos, CEP 52171-900 Recife, PE.
}

E-mail: alisboa@elogica.com.br

${ }^{3}$ Biól., Prof. Titular, Dep. de Botânica, UFSCar, Via Washington Luís, km 235, CEP 13565-905 São Carlos, SP. E-mail: pvmoraes@power.ufscar.br

${ }^{4}$ Eng. Agrôn., Ph.D., Embrapa/Empresa Pernambucana de Pesquisa Agropecuária (IPA), Av. Gen. San Martin, 1371, CEP 50761-000 Recife, PE. E-mail: burity@ipa.br 


\section{INTRODUÇ̃̃O}

A caracterização das condições hídricas dos vegetais está intimamente relacionada com o teor de água nos tecidos e uma maneira de expressar a deficiência de água nesses tecidos, particularmente em folhas, é medir o status de energia da água, ou seu potencial total. Inúmeras variáveis fisiológicas, tais como o nível de ácido abscísico, a fotossíntese líquida, a transpiração, a condutância estomática, entre outros, são correlacionáveis com o potencial total de água na planta ou seus componentes; daí, o interesse na avaliação dessa variável em estudos de relações hídricas de plantas (Hsiao, 1973). Segundo Millar et al. (1971), muitos pesquisadores têm dado preferência às determinações do potencial total da água na planta, porque ele influencia mais diretamente os processos metabólicos que qualquer outro parâmetro de avaliação do déficit hídrico. Entretanto, Acevedo et al. (1979) relatam que as medições do potencial hídrico, isoladamente, não fornecem informações suficientes sobre o impacto do estresse hídrico nos processos fisiológicos medidos pelo conteúdo de água dos tecidos.

Os estudos sobre as trocas gasosas em frutíferas, envolvendo a fixação de $\mathrm{CO}_{2}$, transpiração e resistência difusiva, vêm-se ampliando a partir da década de 80, em trabalhos direcionados para culturas tropicais, como caju e goiaba, murici, cupuaçu, biribá, jenipapo e carambola. O comportamento estomático foi estudado em uva (Miele, 1989) e, especialmente, em variedades de citros (Rieger, 1992). No entanto, com a acerola, esses estudos têm sido escassos.

Diante da importância que a acerola assume no Nordeste, faz-se necessário realizar estudos sobre o comportamento de cultivares desenvolvidas com vistas ao plantio em regime de sequeiro, nos períodos de estiagem impostos em diferentes fases do crescimento, para melhor adequar o manejo da cultura às condições de limitações hídricas.

Este trabalho teve por objetivo estudar o curso diário e sazonal das trocas gasosas, a temperatura foliar e o potencial hídrico foliar da acerola, no campo.

\section{MATERIAL E MÉTODOS}

O trabalho foi realizado no pomar comercial Acerolândia, localizado no município de Paudalho, Zona da Mata Norte do Estado de Pernambuco, a 7055' 58,3" de latitude $\mathrm{Sul}, 35^{\circ} 8^{\prime} 12,8^{\prime \prime}$ de longitude Oeste e $70 \mathrm{~m}$ de altitude.

O município de Paudalho, na mata setentrional pernambucana, apresenta clima quente e úmido, com temperatura e precipitação pluvial média anual de $25^{\circ} \mathrm{C}$ e $1.702,1 \mathrm{~mm}$, respectivamente, e meses chuvosos de março a julho (Fundação de Desenvolvimento Municipal do Interior de Pernambuco, 1994).

As plantas foram propagadas por sementes, com uma considerável variabilidade fenotípica, em relação ao porte, conformação da copa, coloração e morfologia das folhas e dos frutos. O espaçamento utilizado foi de $4 \times 5 \mathrm{~m}$, totalizando aproximadamente 500 plantas adultas ha ${ }^{-1}$. Os tratos culturais necessários à condução do pomar (adubação e poda) são realizadas anualmente. O solo do local, objeto do estudo, é classificado, como Podzólico Amarelo álico (epieutrófico) latossólico A, moderadamente drenado, com textura média/argilosa, e fase floresta subperenifólia com relevo suave ondulado. As características físicas e químicas do horizonte superficial $(0-20 \mathrm{~cm})$ do solo coletado sob a copa das matrizes estudadas foram: densidade: $1,2 \mathrm{~g} \mathrm{~cm}^{-3}$; areia, silte e argila: $53 \%, 18 \%$ e $11 \%$, respectivamente; $\mathrm{pH} 7 ; \mathrm{Na}, \mathrm{K}, \mathrm{Ca}, \mathrm{Mg}$ e Al: $0,22,0,64,9,10,1,30$ e $0,0 \mathrm{mEq} 100 \mathrm{~g}^{-1}$ de solo, respectivamente; P: 268,37 ppm; C e N: 0,25\% e 2,21\%, respectivamente.

Para a consecução deste trabalho foram selecionadas as matrizes UFRPE 7 e UFRPE 8, em função da diferença marcante na morfologia dos frutos (maçã trilobulada e pitanga, respectivamente). Essas plantas, com idade de sete anos, apresentaram, no início do experimento, altura média entre $3,20 \mathrm{~m}$ e $3,80 \mathrm{~m}$, perímetro do caule entre $0,39 \mathrm{~m} \mathrm{e} 0,38 \mathrm{~m}$ e projeção da copa de $4,50 \mathrm{~m} \mathrm{e} \mathrm{4,80} \mathrm{m,}$ respectivamente. No final da estação chuvosa, esses valores foram aumentados para 3,65 $\mathrm{m} \mathrm{e} \mathrm{4,10} \mathrm{m}$ (altura), $0,49 \mathrm{~m}$ e $0,49 \mathrm{~m}$ (perímetro do caule), $5,20 \mathrm{~m}$ e 5,30 $\mathrm{m}$ (projeção da copa), respectivamente.

O conteúdo de água no solo foi determinado nas duas estações (seca e chuvosa) no Laboratório de Física dos Solos da UFRPE, pelo método gravimétrico (Buckman \& Brady, 1974), e os valores, expressos em porcentagem de peso a seco. As avaliações realizadas nesta pesquisa foram as seguintes: 1) Medidas porométricas e do potencial hídrico foliar: foram realizados quatro cursos diários das trocas gasosas do vapor de água, da temperatura foliar e do potencial hídrico da folha, sendo dois na estação seca (15/16 de novembro/94 e 11/12 de dezembro/94) e dois na chuvosa (12/13 de junho/95 e 10/11 de julho/95), além de determinações prévias nos diferentes horários do dia, a fim de se avaliar as variações apresentadas pelas variáveis estudadas e estabelecer o intervalo de medidas. 
Dessa forma, são aqui apresentados e discutidos os resultados dos dois cursos diários dos dias mais claros, relativos à estação seca (11/12 de dezembro/94) e à chuvosa (10/11 de julho/95). De cada planta foram selecionados ramos diretamente expostos à radiação solar, com folhas sadias completamente expandidas, localizadas no terço médio das copas. Nas folhas selecionadas, mediram-se em intervalos de aproximadamente duas horas, no período entre $6 \mathrm{~h}$ e $16 \mathrm{~h}$ a transpiração $(\mathrm{E})$, a resistência difusiva (Rs) e a temperatura foliar (Tf), com auxílio de um porômetro de equilíbrio dinâmico da LICOR, modelo LI-1600. A radiação fotossinteticamente ativa (PAR) foi monitorada por meio de um sensor quântico acoplado ao porômetro. Calculou-se o déficit de pressão de vapor (DPV) segundo Vianello \& Alves (1991). O potencial hídrico foliar $(\Psi \mathrm{w})$ foi determinado utilizando-se uma câmara de pressão de Scholander (Scholander et al., 1965) modelo 3035 da "Soil Moisture Equipment Corp", Santa Bárbara, Califórnia (EUA). Para essas avaliações, foram utilizados os ramos que continham dois a três pares de folhas, e quatro repetições; 2) Capacidade fotossintética a curva da fotossíntese líquida (A) em vista da radiação fotossinteticamente ativa (PAR) foi estabelecida no campo, durante a estação chuvosa (22/julho/1995). As determinações foram realizadas por volta das $9 \mathrm{~h} 30$, com as folhas ligadas ao corpo das plantas. Neste período, a umidade relativa do ar variou de $70 \%$ a $85 \%$, e a temperatura média foliar, entre $25^{\circ} \mathrm{C}$ e $27^{\circ} \mathrm{C}$. Pelo menos três folhas maduras e três folhas jovens de cada matriz foram medidas previamente. Uma das folhas de cada estádio de maturação, que apresentou a maior taxa de fotossíntese líquida, foi selecionada para realizar a curva de fotossíntese versus radiação. Foram feitos três a quatro registros por folha, após a fotossíntese líquida ter atingido valor constante. Os dados foram obtidos com um analisador portátil para medir fotossíntese por infravermelho, ligado a uma câmara foliar tipo Parkinson, modelo PLC- N, com $12 \mathrm{~cm}^{2}$ de área de exposição foliar à radiação solar e com volume interno de, aproximadamente, $12 \mathrm{~cm}^{3}$; uma unidade digital de ar para suprimento de fluxo constante. Os dados foram ajustados mediante uma expressão matemática do tipo não-linear, e a equação escolhida para ajustar os pares de pontos fotossíntese versus radiação foi extraída do trabalho de Pereira Netto \& Hay (1986), e modificada pela introdução do ponto de compensação à luz (PCL). Para análise comparativa entre as médias das demais variáveis, utilizou-se ANOVA (análise de variância), e para separação, o teste de Tukey, além da terminação de correlação linear simples, para estimar o grau de relação entre os pares de variáveis.

\section{RESULTADOS E DISCUSSÃo}

\section{Curso diário das trocas gasosas do vapor de água, da temperatura foliar e do potencial hídrico da folha}

A radiação fotossinteticamente ativa apresentou variações diárias de 81 a $1.528 \mu \mathrm{mol} \mathrm{m}^{-2} \mathrm{~s}^{-1}$ na estação seca e de 18 a $1.648 \mu \mathrm{mol} \mathrm{m}{ }^{-2} \mathrm{~s}^{-1}$ na chuvosa. Os maiores valores na estação seca foram registrados às $14 \mathrm{~h}$, e na chuvosa, às $10 \mathrm{~h}$. Ressalta-se que, na Zona da Mata, geralmente, não são comuns dias inteiros totalmente sem nuvens, mesmo durante a estação seca.

A temperatura do ar na estação seca durante o curso do dia variou de $24,10^{\circ} \mathrm{C}$ a $32,88^{\circ} \mathrm{C}$. Na estação chuvosa, os valores situaram-se entre $22,20^{\circ} \mathrm{C}$ e $30,28^{\circ} \mathrm{C}$, e os máximos foram observados às $12 \mathrm{~h}$, em ambas as estações.

A evolução da umidade relativa mostra uma depressão no decorrer da manhã, culminando com os baixos valores ao meio do dia e uma recuperação a partir das $14 \mathrm{~h}$. Os valores registrados na época seca situaram-se entre $36,78 \%$ e $74,18 \%$, e na época chuvosa, entre $61,65 \%$ e $91,95 \%$.

O déficit de pressão de vapor (DPV) variou em função da sazonalidade e do curso diário. Os valores médios foram de 0,75 a $3,25 \mathrm{kPa}$ na estação seca e de 0,20 a $1,80 \mathrm{kPa}$, na chuvosa (Tabela 1), e os maiores déficits ocorreram nas horas mais quentes do dia.

As matrizes UFRPE 7 e UFRPE 8 mostraram padrões de comportamento diferentes nas duas estações. A análise do andamento diário da transpiração demonstrou que as duas matrizes possuem razoável regulação estomática, pois quando expostas a radiações fotossinteticamente ativas acima de $400 \mu \mathrm{mol} \mathrm{m} \mathrm{m}^{-2} \mathrm{~s}^{-1}$ associadas a umidades relativas abaixo de $50 \%$, já apresentam restrições para a perda de água, comportamento verificado mais acentuadamente na estação seca. Nessa época, a mais baixa temperatura (aproximadamente $24^{\circ} \mathrm{C}$ ) matinal e a umidade relativa em torno de $80 \%$ no início do dia amenizam os rigores do baixo potencial hídrico do solo, permitindo maior troca gasosa da planta com o ambiente nesse momento. No entanto, nessa época, ao analisar-se o curso diário da transpiração das duas matrizes, verificou-se que elas apresentavam comportamentos distintos, ou seja: a UFRPE 7 mantém o controle estomático durante todo o dia, sem muita 
variação, enquanto a UFRPE 8 transpira mais no início da manhã e final da tarde, apresentando decréscimos graduais até às $14 \mathrm{~h}$, quando o PAR se apresenta mais intenso.

Na estação chuvosa, observa-se maior variação na transpiração da UFRPE 7. Valores altos no início da manhã $\left(5,5 \mathrm{mmol} \mathrm{m} \mathrm{m}^{-2} \mathrm{~s}^{-1}\right)$ e final da tarde $\left(4,0 \mathrm{mmol} \mathrm{m}^{-2} \mathrm{~s}^{-1}\right)$, decaindo para mais de $50 \%$ no período das $8 \mathrm{~h}$ às $14 \mathrm{~h}$. Por outro lado, a UFRPE 8 mantém a taxa transpiratória em torno de $3,50 \mathrm{mmol} \mathrm{m}^{-2} \mathrm{~s}^{-1}$ a $3,75 \mathrm{mmol} \mathrm{m}^{-2} \mathrm{~s}^{-1}$ durante todo o dia, exceto às $8 \mathrm{~h}$, reduzindo para $3 \mathrm{mmol} \mathrm{m}^{-2} \mathrm{~s}^{-1}$, o que coincide com o mais alto PAR registrado nesse dia $\left(980 \mu \mathrm{mol} \mathrm{m} \mathrm{m}^{-2} \mathrm{~s}^{-1}\right)$. Os valores da transpiração obtidos nesse período situam-se próximo aos de Citrus sinensis $\left(3,0 \mathrm{mmol} \mathrm{m}{ }^{-2} \mathrm{~s}^{-1}\right.$ a 4,4 $\mathrm{mmol} \mathrm{m}^{-2} \mathrm{~s}^{-1}$ ), encontrados por Machado et al. (1994), superiores aos de Theobroma cacao $\left(2,0 \mathrm{mmol} \mathrm{m} \mathrm{m}^{-2} \mathrm{~s}^{-1}\right)$, verificados por Galyuon et al. (1996b), e inferiores aos de banana, Musa AAA (10,0 mmol m-2 $\mathrm{s}^{-1}$ a $\left.18,0 \mathrm{mmol} \mathrm{m}^{-2} \mathrm{~s}^{-1}\right)$, segundo Eckstein \& Robinson (1996)

Com relação à estação seca, os menores valores da transpiração foram verificados às $14 \mathrm{~h}$ com o fechamento parcial dos estômatos $\left(0,60 \mathrm{mmol} \mathrm{m}^{-2} \mathrm{~s}^{-1}\right)$, enquanto os maiores foram registrados às $6 \mathrm{~h}$ $\left(1,26 \mathrm{mmol} \mathrm{m}^{-2} \mathrm{~s}^{-1}\right)$ e às $16 \mathrm{~h}\left(1,25 \mathrm{mmol} \mathrm{m}^{-2} \mathrm{~s}^{-1}\right)$.

Independentemente da sazonalidade, as matrizes apresentaram diferenças significativas entre si e em função do curso diário (Tabela 2). Na estação chuvosa, a matriz UFRPE 7 transpirou, durante o dia, uma média de $2,75 \mathrm{mmol} \mathrm{m}^{-2} \mathrm{~s}^{-1}$, enquanto a UFRPE 8 transpirou $3,58 \mathrm{mmol} \mathrm{m}^{-2} \mathrm{~s}^{-1}$. Na estação seca, esses valores foram reduzidos para $0,78 \mathrm{mmol} \mathrm{m}^{-2} \mathrm{~s}^{-1} \mathrm{e}$ $1,34 \mathrm{mmol} \mathrm{m}^{-2} \mathrm{~s}^{-1}$, respectivamente. Com isso, observamos que a UFRPE 8 conseguiu manter as trocas gasosas com mais eficiência que a UFRPE 7, mesmo na época de restrição de disponibilidade hídrica do solo e altas demandas evaporativas.

As Figs. 1 e 2 ilustram o andamento diário da resistência difusiva das matrizes de acerola nas estações seca e chuvosa. A sazonalidade teve grande influência na resistência difusiva das plantas estudadas, a qual mostrou-se bem mais acentuada na estação seca que na chuvosa, com valores médios diários de $9,82 \mathrm{~s} \mathrm{~cm}^{-1}$ e $1,62 \mathrm{~s} \mathrm{~cm}^{-1}$, respectivamente, e os máximos da estação seca $18,37 \mathrm{~s} \mathrm{~cm}^{-1}$ (Tabela 2) foram registrados às $14 \mathrm{~h}$, coincidindo com os mais altos valores do PAR e do DPV. A matriz UFRPE 7 apresentou os maiores valores médios diários de resistência difusiva, tanto na estação seca $\left(10,70 \mathrm{~s} \mathrm{~cm}^{-1}\right)$ quanto na chuvosa $\left(2,33 \mathrm{~s} \mathrm{~cm}^{-1}\right)$, quando comparada com a UFRPE 8, cujos valores foram de $8,95 \mathrm{~s} \mathrm{~cm}^{-1}$ e $0,91 \mathrm{~s} \mathrm{~cm}^{-1}$, respectivamente.

Avaliando a condutância estomática em três espécies de cerradão nas estações seca e chuvosa, Perez (1984) verificou valores médios situados entre $0,04 \mathrm{~cm} \mathrm{~s}^{-1}$ e $0,02 \mathrm{~cm} \mathrm{~s}^{-1}$ na estação seca, e uma variação de $0,17 \mathrm{~cm} \mathrm{~s}^{-1}$ a $0,09 \mathrm{~cm} \mathrm{~s}^{-1}$ na estação chuvosa. Transformando esses dados para o seu inverso - a resistência difusiva - os valores foram de 25,0 a

TABELA 1. Valores médios \pm desvio-padrão do déficit de pressão de vapor (DPV) de água (kPa) durante as estações seca e chuvosa, por ocasião de avaliações porométricas e do potencial hídrico foliar, em duas matrizes de acerola cultivadas sob condições de campo, nas estações seca e chuvosa. Médias de quatro repetições. Acerolândia, Paudalho, PE.

\begin{tabular}{cccccc}
\hline \multirow{2}{*}{$\begin{array}{c}\text { Tempo } \\
(\mathrm{h})\end{array}$} & \multicolumn{3}{c}{ Seca } & & \multicolumn{2}{c}{ Chuvosa } \\
\cline { 2 - 3 } \cline { 5 - 6 } & UFRPE 7 & UFRPE 8 & & UFRPE 7 & UFRPE 8 \\
\hline & $0,82 \pm 0,018$ & $0,81 \pm 0,041$ & & $0,22 \pm 0,019$ & $0,26 \pm 0,014$ \\
6 & $1,79 \pm 0,017$ & $2,15 \pm 0,047$ & & $0,71 \pm 0,154$ & $0,55 \pm 0,028$ \\
10 & $2,75 \pm 0,099$ & $2,66 \pm 0,028$ & & $1,07 \pm 0,046$ & $1,03 \pm 0,031$ \\
12 & $3,12 \pm 0,063$ & $2,96 \pm 0,012$ & & $1,28 \pm 0,180$ & $1,65 \pm 0,071$ \\
14 & $2,77 \pm 0,050$ & $2,77 \pm 0,050$ & & $1,18 \pm 0,097$ & $1,45 \pm 0,070$ \\
16 & $1,69 \pm 0,035$ & $1,48 \pm 0,013$ & & $0,84 \pm 0,033$ & $0,99 \pm 0,028$ \\
\hline
\end{tabular}


$50,0 \mathrm{~cm} \mathrm{~s}^{-1}$ e 5,8 a $11,1 \mathrm{~cm} \mathrm{~s}^{-1}$, respectivamente. Comparando com os resultados obtidos nas matrizes de acerola, observa-se que elas apresentaram valores médios inferiores, tanto na estação seca $(8,95$ a $\left.10,70 \mathrm{~cm} \mathrm{~s}^{-1}\right)$ quanto na chuvosa $\left(0,91 \mathrm{a} 2,33 \mathrm{~cm} \mathrm{~s}^{-1}\right)$, possibilitando dessa forma, maiores trocas gasosas.

O padrão de comportamento das curvas da resistência difusiva da UFRPE 7 foi similar ao registrado no tocante ao PAR e temperatura foliar, seguindo a mesma tendência, com mais evidência na estação seca.

Os valores do potencial hídrico foliar variaram de acordo com a hora do dia, em ambas as matrizes, independentemente da sazonalidade. Os valores mínimos ocorreram na época seca, sendo a UFRPE 8, nesta estação, a que apresentou os menores valores, e os maiores, na chuvosa (Figs. 1 e 2). Os mínimos registrados situaram-se entre $-3,37 \mathrm{MPa}$ (UFRPE 7) e -4,32 MPa (UFRPE 8) durante a estação seca, e entre -1,97 MPa (UFRPE 8) e -2,22 MPa (UFRPE 7) na estação chuvosa. Segundo Farquhar \& Sharkey (1982), mudanças na taxa transpiratória podem causar mudanças na temperatura foliar, bem como no potencial hídrico foliar.

Os decréscimos ocorridos no potencial hídrico foliar, bem como os aumentos verificados na resis- tência difusiva das matrizes na estação seca, estão relacionados com o teor de umidade do solo (Tabela 3 ), com os aumentos da temperatura foliar (Figs. 1 e 2), associados a um maior déficit de pressão de vapor de água ocorrido na referida estação (Tabela 2), como foi demonstrado em citros (Kriedemann, 1971; Vu \& Yelenosky, 1988). Por outro lado, os valores do $\Psi_{\mathrm{w}}$ podem variar, de acordo com o estádio fenológico da cultura, da variedade, da disponibilidade hídrica do solo, do déficit de pressão de vapor, e do horário em que o mesmo é registrado.

$\mathrm{O} \Psi_{\mathrm{w}}$ é uma medida do status de água na planta. Uma elevação da Rs e conseqüente redução da E pode promover um ligeiro aumento do $\Psi_{\mathrm{w}}$. Segundo Larcher (1975), as espécies arbóreas adultas têm grande superfície evaporante, e quando há um suprimento adequado de água, transpiram livremente, podendo ocasionar valores mais negativos de $\Psi_{\mathrm{w}}$. Entretanto, muitas espécies herbáceas que não apresentam sistema radicular eficiente podem apresentar um $\Psi_{\mathrm{w}}$ mais elevado, em conseqüência de uma condutância estomática menor, ou de uma maior resistência estomática (Perez \& Moraes, 1991).

A temperatura das folhas da UFRPE 7, durante a estação seca, esteve acima da temperatura do ar, em todos os intervalos de medidas, com uma diferença

TABELA 2. Valores médios obtidos para transpiração (E), resistência difusiva ao vapor de água (Rs), temperatura foliar (Tf) e potencial da água da folha (Yw) em duas matrizes de acerola (UFRPE 7 e UFRPE 8), cultivadas sob condições de campo, nas estações seca e chuvosa'.

\begin{tabular}{|c|c|c|c|c|}
\hline Fatores & 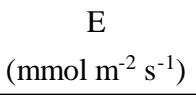 & $\begin{array}{c}\mathrm{Rs} \\
\left(\mathrm{s} \mathrm{cm}^{-1}\right)\end{array}$ & $\begin{array}{l}\mathrm{Tf} \\
\left({ }^{\circ} \mathrm{C}\right)\end{array}$ & $\begin{array}{c}\Psi \mathrm{w} \\
(\mathrm{MPa})\end{array}$ \\
\hline \multicolumn{5}{|l|}{ Matrizes } \\
\hline UFRPE 7 & $1,77 b$ & $6,52 \mathrm{a}$ & $29,34 \mathrm{a}$ & $-1,84 a$ \\
\hline UFRPE 8 & $2,46 a$ & $4,93 b$ & $28,44 \mathrm{~b}$ & $-2,08 b$ \\
\hline \multicolumn{5}{|l|}{ Estações } \\
\hline Seca & $1,06 b$ & $9,82 \mathrm{a}$ & $31,16 a$ & $-2,66 b$ \\
\hline Chuvosa & $3,16 \mathrm{a}$ & $1,62 b$ & $26,91 b$ & $-1,25 \mathrm{a}$ \\
\hline \multicolumn{5}{|l|}{ Estação seca } \\
\hline UFRPE 7 & $0,78 b$ & $10,69 a$ & $31,85 \mathrm{a}$ & $-2,33 a$ \\
\hline UFRPE 8 & $1,34 \mathrm{a}$ & $8,95 \mathrm{~b}$ & $30,48 b$ & $-2,99 b$ \\
\hline \multicolumn{5}{|l|}{ Estação chuvosa } \\
\hline UFRPE 7 & $2,75 b$ & $2,33 \mathrm{a}$ & $27,43 a$ & $-1,35 b$ \\
\hline UFRPE 8 & $3,58 \mathrm{a}$ & $0,91 b$ & $26,39 b$ & $-1,16 a$ \\
\hline
\end{tabular}


máxima de $3,75^{\circ} \mathrm{C}$, verificada ao meio-dia, e mínima $\left(0,28^{\circ} \mathrm{C}\right)$, no final da tarde. Tal comportamento também foi verificado na estação chuvosa, nos horários de $8 \mathrm{~h}, 10 \mathrm{~h}$ e $14 \mathrm{~h}$, com diferenças de $0,55^{\circ} \mathrm{C}, 2,85^{\circ} \mathrm{Ce}$ $3,13^{\circ} \mathrm{C}$, respectivamente. Para a UFRPE 8 , na estação seca a temperatura foliar foi superior à do ar a partir do meio-dia, sendo registrada a diferença máxima $\left(2,6^{\circ} \mathrm{C}\right)$, às $14 \mathrm{~h}$. Na estação chuvosa, apenas no ho-

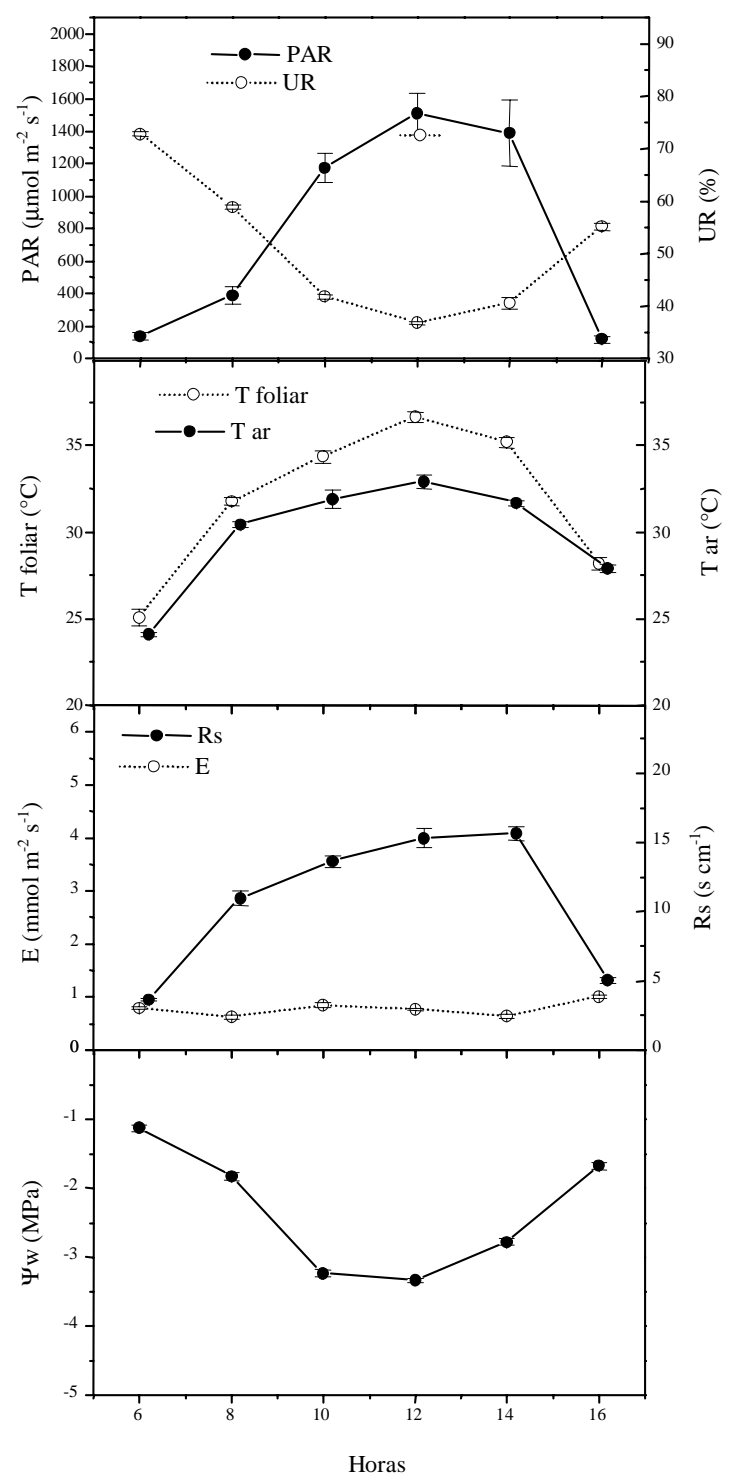

Estação seca

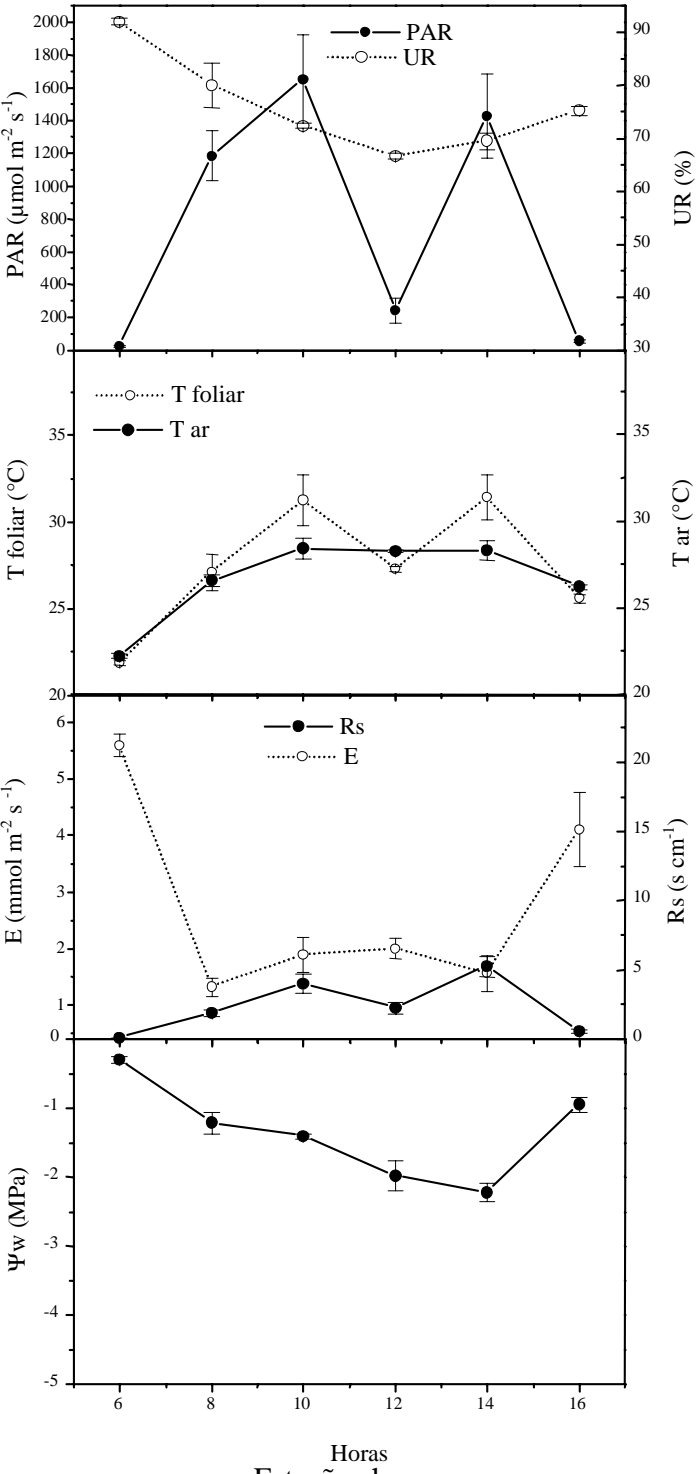

Estação chuvosa

FIG. 1. Cursos diários da radiação fotossinteticamente ativa (PAR), umidade relativa do ar (UR), temperatura da folha ( $T$ foliar), temperatura do ar ( $T$ ar), transpiração (E), resistência difusiva (Rs) e potencial hídrico foliar (Yw), na matriz UFRPE 7, durante as estações seca (11/12/94) e chuvosa (10/07/95). As linhas representam valores médios de pelo menos quatro determinações, e as barras, o desvio-padrão. 
rário das $8 \mathrm{~h}$ a temperatura da folha foi maior que a do ar, com uma diferença de $1,13^{\circ} \mathrm{C}$ (Figs. 1 e 2). Resultados semelhantes foram verificados para folhas de laranjeira (Machado et al., 1994) e de acerola, durante a estação seca (Nogueira \& Moraes, 1997). Os primeiros autores relatam que as temperaturas das folhas expostas à radiação solar estiveram entre $1^{\circ} \mathrm{C}$ (9h) e $5^{\circ} \mathrm{C}(13 \mathrm{~h})$, enquanto os segundos verificaram diferenças de até $4^{\circ} \mathrm{C}$. Essa variação é comum em algumas espécies cultivadas, e fundamenta-se no

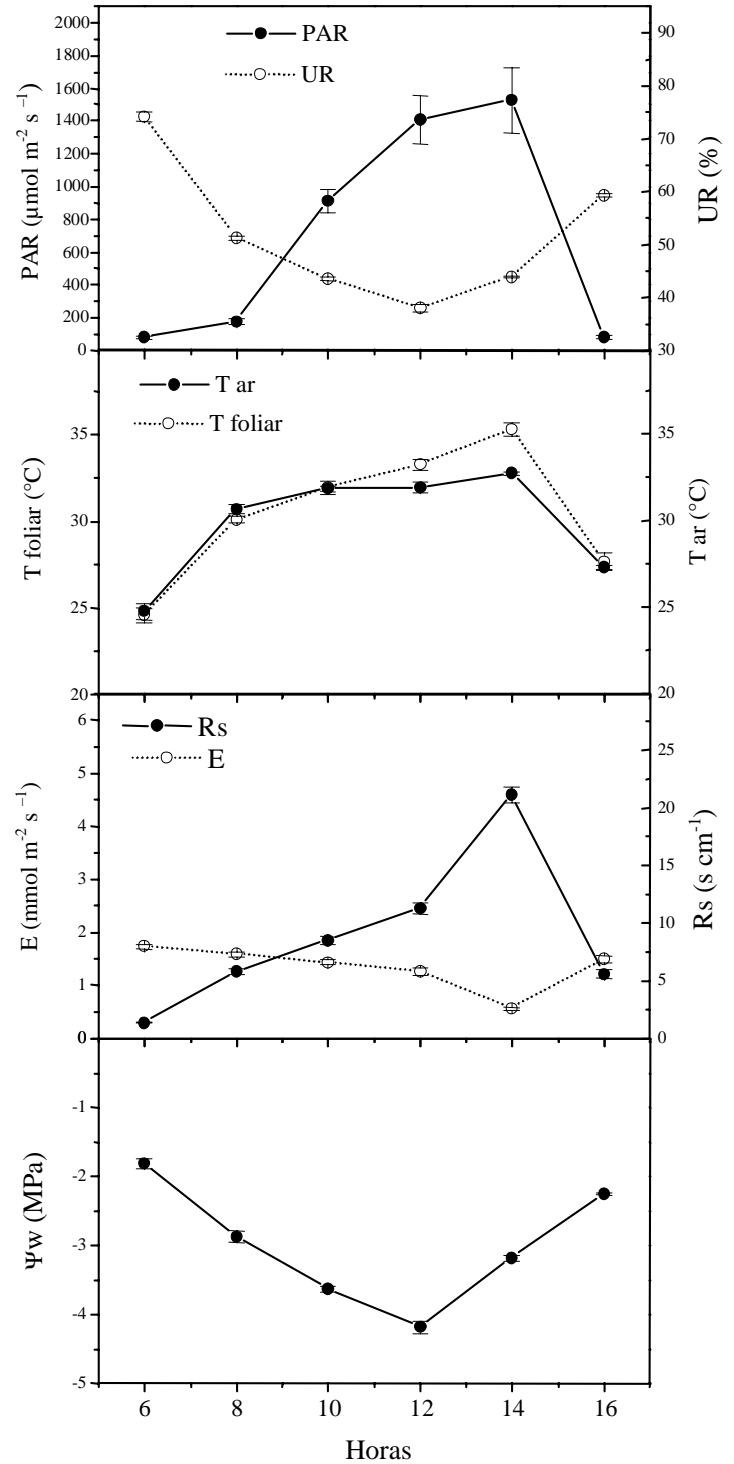

Estação seca

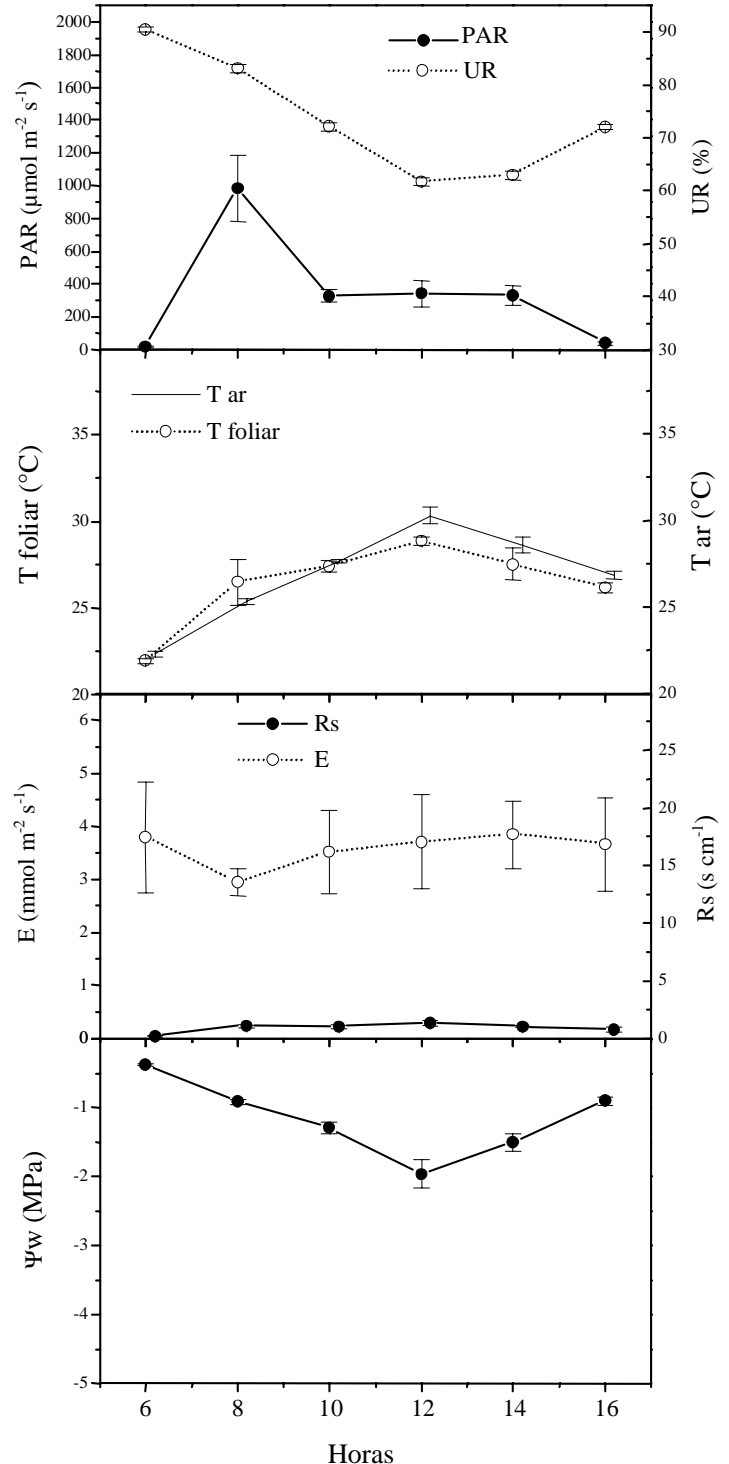

Estação chuvosa

FIG. 2. Cursos diários da radiação fotossinteticamente ativa (PAR), umidade relativa do ar (UR), temperatura da folha ( $T$ foliar), temperatura do ar ( $T$ ar), transpiração $(E)$, resistência difusiva (Rs) e potencial hídrico foliar (Y) na matriz UFRPE 8, durante as estações seca (12/12/94) e chuvosa (10/07/95). As linhas representam valores médios de pelo menos quatro determinações, e as barras, o desvio-padrão. 
estado hídrico delas, no comportamento estomático e na perda de calor latente através da transpiração. Tais diferenças normalmente diminuem no início da manhã e no final da tarde (Salinas et al., 1996).

Com relação às análises de regressão linear simples associando valores do déficit de pressão de vapor de água entre a folha e o ar (DPV) aos da transpiração, resistência difusiva, potencial hídrico e temperatura foliar das matrizes de acerola, nas estações seca e chuvosa (Tabela 4), a transpiração da

TABELA 3. Valores médios e desvios-padrões da umidade $(\%)$ do solo de duas matrizes de acerola (UFRPE 7 e UFRPE 8) do pomar comercial Acerolândia (município de Paudalho, PE), durante as estações seca $\mathrm{e}$ chuvosa. As amostras foram retiradas de sob a copa das matrizes a uma profundidade de $20 \mathrm{~cm}$. Médias de cinco repetições.

Matrizes $\quad$ Estação seca (dez./94) $\quad$ Estação chuvosa (jul./95)

\begin{tabular}{lll}
\hline UFRPE 7 & $6,42 \pm 0,337$ & $29,34 \pm 0,386$ \\
UFRPE 8 & $6,76 \pm 0,453$ & $29,27 \pm 0,488$ \\
\hline
\end{tabular}

matriz UFRPE 7 esteve associada ao DPV segundo uma exponencial negativa $\left(\mathrm{r}=-0,71^{*}\right)$ durante a estação chuvosa, enquanto na UFRPE 8 este mesmo comportamento foi verificado apenas na estação seca $\left(\mathrm{r}=-0,65^{*}\right)$. A baixa resposta observada nesta última matriz na condição de adequado suprimento hídrico do solo, pode ser devido aos valores pouco elevados do DPV durante os períodos de chuva. O efeito direto do DPV sobre a abertura estomática foi descrito em outras espécies, tais como laranja e limão (Kriedemann, 1968) e banana (Eckstein \& Robinson, 1996).

Vários estudos têm demonstrado uma boa relação entre resistência estomática e o déficit de saturação de vapor de água do ar (Black \& Squire, 1979). Schulze et al. (1972) consideram que baixa umidade do ar atua no fechamento estomático, que a alta umidade conduz à abertura, e que os estômatos funcionam como o principal sensor de umidade.

O potencial hídrico foliar correlacionou-se negativamente com o DPV, tanto na estação seca quanto na chuvosa. As variações do déficit de pressão de vapor de água entre a folha e o ar explicaram, na matriz UFRPE $7,95 \%$ e $88 \%$ das variações do potencial hídrico nas estações seca e chuvosa, respecti-

TABELA 4. Regressão linear e valores de correlação (r) entre o déficit de pressão de vapor (DPV) de água (kPa) e a transpiração (E), a resistência difusiva (Rs), o potencial hídrico foliar ( $\Psi$ f) e a temperatura da folha (Tf), em duas matrizes de acerola (UFRPE 7 e UFRPE 8) cultivadas sob condições de campo, nas estações seca e chuvosa. Acerolândia, Paudalho (PE).

\begin{tabular}{|c|c|c|c|c|}
\hline \multirow[t]{2}{*}{ Variáveis } & \multicolumn{2}{|c|}{ Estação seca } & \multicolumn{2}{|c|}{ Estação chuvosa } \\
\hline & UFRPE 7 & UFRPE 8 & UFRPE 7 & UFRPE 8 \\
\hline DPV x E & $\begin{array}{c}y=0,85-0,03 x \\
\mathbf{r}=-0,19^{\text {ns }}\end{array}$ & $\begin{array}{c}y=2,03-0,32 x \\
\mathbf{r}=-0,65^{*}\end{array}$ & $\begin{array}{c}y=5,51-3,14 x \\
\mathbf{r}=-0,71^{*}\end{array}$ & $\begin{array}{c}y=3,28-0,29 x \\
\mathbf{r}=-0,17^{\mathrm{ns}}\end{array}$ \\
\hline DPV x Rs & $\begin{array}{c}\mathrm{y}=-1,15+5,48 \mathrm{x} \\
\mathbf{r}=0,93^{* *}\end{array}$ & $\begin{array}{c}y=-4,13+6,13 x \\
\mathbf{r}=0,75^{*}\end{array}$ & $\begin{array}{c}y=-0,86+3,63 x \\
\mathbf{r}=-0,71^{*}\end{array}$ & $\begin{array}{c}y=-0,32+0,59 x \\
\mathbf{r}=-0,73^{*}\end{array}$ \\
\hline DPV x $\Psi f$ & $\begin{array}{c}y=-0,32-0,95 x \\
\mathbf{r}=-0,95^{* *}\end{array}$ & $\begin{array}{c}y=-0,89-0,98 x \\
\mathbf{r}=-0,94 * *\end{array}$ & $\begin{array}{c}y=-0,04-1,57 x \\
\mathbf{r}=-0,88^{* *}\end{array}$ & $\begin{array}{c}y=-0,19-0,98 x \\
\mathbf{r}=-0,92 * *\end{array}$ \\
\hline DPV x Tf & $\begin{array}{c}\mathrm{y}=21,23+4,91 \mathrm{x} \\
\mathbf{r}=0,97^{* *}\end{array}$ & $\begin{array}{c}\mathrm{y}=20,94+4,47 \mathrm{x} \\
\mathbf{r}=0,96^{* *}\end{array}$ & $\begin{array}{c}\mathrm{y}=21,19+7,08 \mathrm{x} \\
\mathbf{r}=0,76^{*}\end{array}$ & $\begin{array}{c}\mathrm{y}=22,53+3,92 \mathrm{x} \\
\mathbf{r}=0,83^{* *}\end{array}$ \\
\hline
\end{tabular}

ns,$*$ e ** Não-significativo e significativo a $5 \%$ e $1 \%$ de probabilidade. 
vamente. Na UFRPE 8 , esses porcentuais foram de $94 \%$ e $92 \%$.

Os efeitos do DPV sobre a temperatura foliar das matrizes estudadas foram mais acentuados na estação seca, quando o solo apresentava-se com baixas umidades (entre $6,42 \%$ e $6,76 \%$ ). Neste período, o DPV explicou $97 \%$ das variações da temperatura foliar quanto à matriz UFRPE $7, \mathrm{e} 96 \%$ quanto à UFRPE 8.

\section{Resposta da fotossíntese à intensidade luminosa}

Para evidenciar a resposta fotossintética em folhas jovens e adultas das duas matrizes em relação à variação da intensidade luminosa, foram plotados os valores das taxas de fotossíntese contra os valores da radiação fotossinteticamente ativa (PAR). Através do ajustamento das curvas (Figs. 3 e 4), pode-se estimar a taxa de fotossíntese máxima (Amax) num dia de boa disponibilidade hídrica do solo, ou seja, durante a estação chuvosa.

A matriz UFRPE 8 apresentou maior capacidade fotossintética que a UFRPE 7, independentemente do estádio de maturação das folhas. Por outro lado, Amax foi maior nas folhas maduras. Para essas últimas, os valores encontrados foram de $12,42 \mu \mathrm{mol} \mathrm{m}^{-2} \mathrm{~s}^{-1} \mathrm{e}$ $9,63 \mu \mathrm{mol} \mathrm{m}^{-2} \mathrm{~s}^{-1}$, respectivamente. O ponto de compensação luminoso (PCL) para as folhas maduras da matriz UFRPE 8 foi de $70,21 \mu \mathrm{mol} \mathrm{m}^{-2} \mathrm{~s}^{-1}$, apresentando rendimento quântico de 0,032 . Na UFRPE 7, esses valores foram de $105,15 \mu \mathrm{mol} \mathrm{m}^{-2} \mathrm{~s}^{-1} \mathrm{e}$ de 0,018 , respectivamente. Os valores de Amax e PCL, em folhas jovens das duas matrizes, foram de $6,38 \mu \mathrm{mol} \mathrm{m}^{-2} \mathrm{~s}^{-1} \mathrm{e}$ 0,023 (UFRPE 8), e de $5,99 \mu \mathrm{mol} \mathrm{m}^{-2} \mathrm{~s}^{-1}$ e 0,022 (UFRPE 7), respectivamente.

Os valores de radiação fotossinteticamente ativa que saturam em pelo menos $90 \%$ a fotossíntese líquida, foram aproximados nas folhas maduras das duas matrizes (em torno de $1.300 \mu \mathrm{mol} \mathrm{m}^{-2} \mathrm{~s}^{-1}$ ). Em algumas espécies lenhosas do cerrado, esses variaram de $600 \mu \mathrm{mol} \mathrm{m}^{-2} \mathrm{~s}^{-1}$ a $899 \mu \mathrm{mol} \mathrm{m}^{-2} \mathrm{~s}^{-1}$ (Prado, 1994).

Os valores observados nas matrizes em estudo encontram-se bem superiores aos referidos acima em plantas de sol, bem como aos verificados por Kanno (1993) nas três espécies de cerrado: Anacardium nanum $\left(67,22 \mu \mathrm{mol} \mathrm{m}^{-2} \mathrm{~s}^{-1}\right)$, Eriotheca gracilipes (39,64 $\left.\mu \mathrm{mol} \mathrm{m} \mathrm{m}^{-2} \mathrm{~s}^{-1}\right)$ Andirahumilis (36,77 $\left.\mu \mathrm{mol} \mathrm{m}^{-2} \mathrm{~s}^{-1}\right)$.
Em indivíduos jovens de Stryphnodendron adstringens, Rocha \& Moraes (1997) registraram um PCL de $59,0 \mu \mathrm{mol} \mathrm{m}^{-2} \mathrm{~s}^{-1}$.

As taxas máximas de fotossíntese obtidas nas matrizes se incluem dentro dos limites verificados em plantas lenhosas decíduas $\left(6,5\right.$ a $\left.16 \mu \mathrm{mol} \mathrm{m}^{-2} \mathrm{~s}^{-1}\right)$ e árvores decíduas frutíferas $\left(6,5 \mathrm{a} 20 \mu \mathrm{mol} \mathrm{m}^{-2} \mathrm{~s}^{-1}\right)$, segundo Körner et al. (1979). Em relação às aceroleiras do presente estudo, os mesmos situaram-se próximos aos de Citrus sinensis $\left(9 \mu \mathrm{mol} \mathrm{m}^{-2} \mathrm{~s}^{-1}\right.$ a $\left.12 \mu \mathrm{mol} \mathrm{m}^{-2} \mathrm{~s}^{-1}\right)$ encontrados por
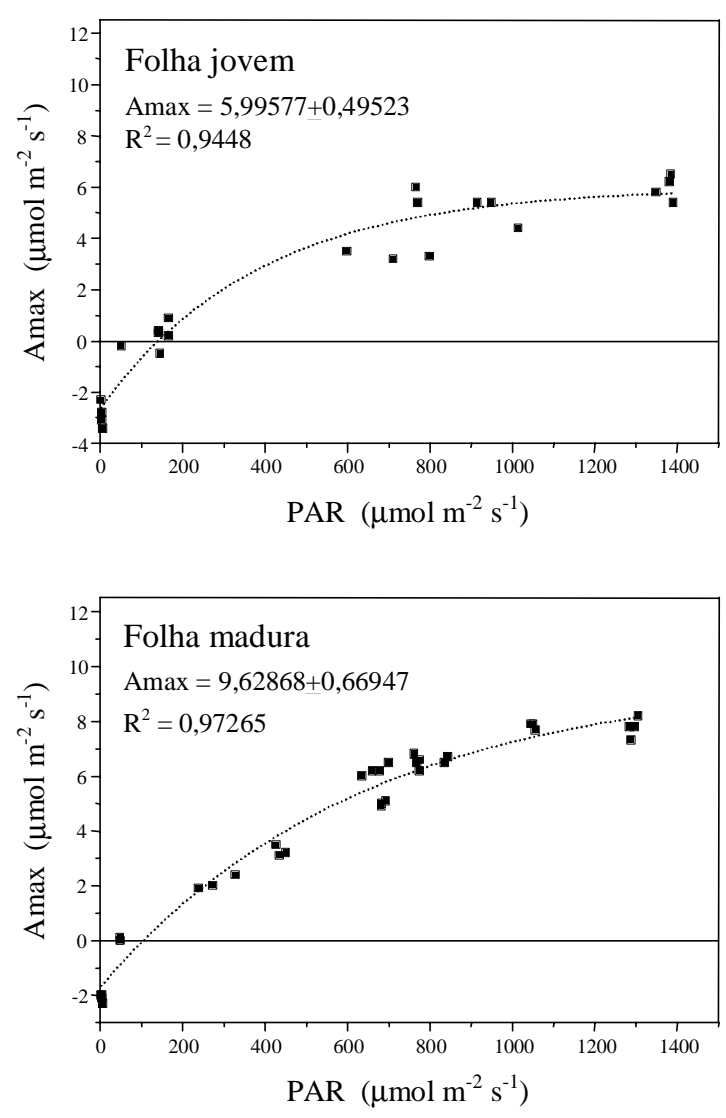

FIG. 3. Fotossíntese líquida expressa em área (Amax) em função da radiação fotossinteticamente ativa (PAR) em folhas jovens e maduras de Malpighia emarginata D.C., Matriz UFRPE 7, cultivada em pomar comercial. Estação chuvosa (julho/95). Acerolândia, Paudalho (PE). 

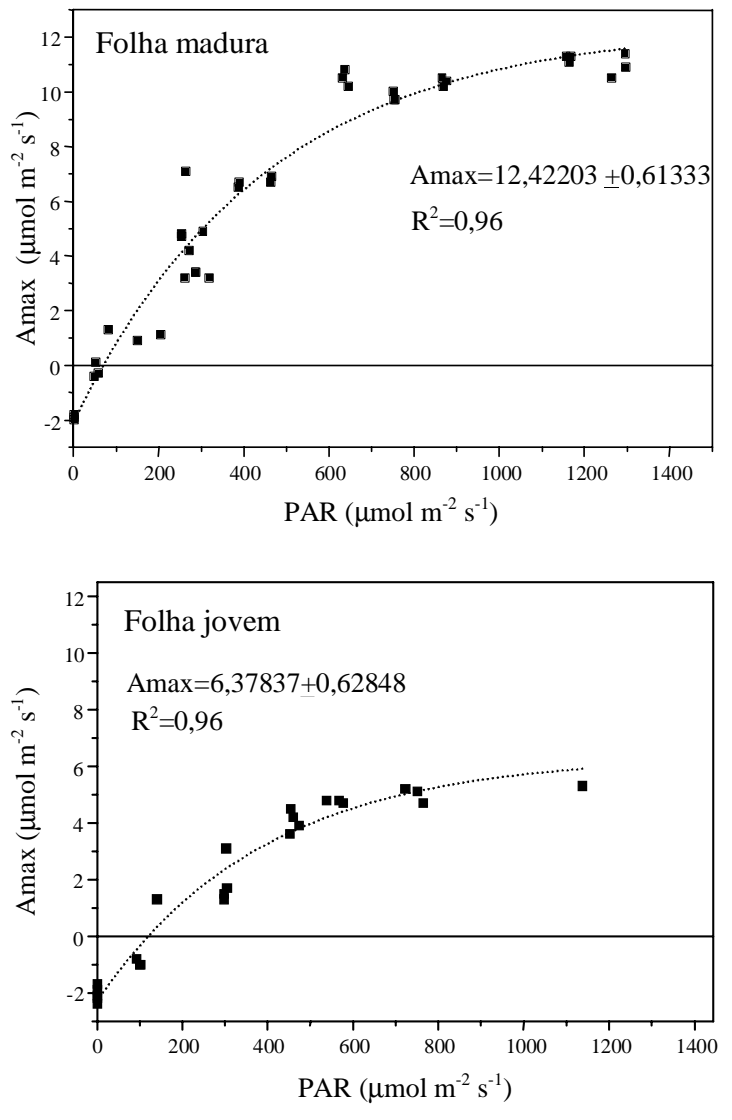

FIG. 4. Fotossíntese líquida expressa em área (Amax) em função da radiação fotossinteticamente ativa (PAR) em folhas jovens e maduras de Malpighia emarginata D.C., Matriz UFRPE 8, cultivada em pomar comercial. Estação chuvosa (julho/95). Acerolândia, Paudalho (PE).

Machado et al. (1994); superiores aos valores de Theobroma cacao $\left(3,5 \mu \mathrm{mol} \mathrm{m}^{-2} \mathrm{~s}^{-1}\right)$ e Vitis vinifera $\left(3,97 \mu \mathrm{mol} \mathrm{m}{ }^{-2} \mathrm{~s}^{-1}\right.$ a $\left.5,74 \mu \mathrm{mol} \mathrm{m} \mathrm{m}^{-2} \mathrm{~s}^{-1}\right)$ verificados por Galyuon et al. (1996a) e Miele (1989), respectivamente, e inferiores aos de banana, Musa AAA $\left(15 \mu \mathrm{mol} \mathrm{m}^{-2} \mathrm{~s}^{-1}\right.$ a $43 \mu \mathrm{mol} \mathrm{m}^{-2} \mathrm{~s}^{-1}$ ), segundo Eckstein \& Robinson (1996).

\section{CONCLUSÕES}

1. O estresse hídrico atua na regulação das relações hídricas das duas matrizes, restringindo a transpiração, aumentando a resistência difusiva e reduzindo o potencial hídrico da folhas.

2. O déficit de pressão de vapor reduz significativamente o potencial hídrico foliar das duas matrizes, e este efeito é pronunciado tanto na estação seca quanto na chuvosa.

3. As respostas dos estômatos à demanda evaporativa da atmosfera e à disponibilidade hídrica do solo mostram que o fechamento estomático é um mecanismo sensível e destinado a reduzir o déficit hídrico nas plantas estudadas, para suportar determinados períodos de seca.

4. O aumento da resistência difusiva das duas matrizes é acompanhado pelo aumento da temperatura foliar, sendo bem mais evidenciado na estação seca.

5. A matriz UFRPE 8 é mais adaptada a períodos de estiagem do que a UFRPE 7.

\section{AGRADECIMENTOS}

Ao Sr. Alcindo Lacerda, proprietário do pomar Acerolândia, pela atenção dispensada durante a realização deste trabalho, e ao pesquisador da Empresa Pernambucana de Pesquisa Agropecuária (IPA), Odemar Vicente dos Reis, pela orientação nas análises estatísticas dos dados.

\section{REFERÊNCIAS}

ACEVEDO, E.T.C.; FERERES, E.; HSIAO, T.C.; HENDERSON, D.W. Diurnal growth trends, water potential and osmotic adjustment of maize and sorghum leaves in the field. Plant Physiology, Rockville, v.64, p.476-480, 1979

BLACK, C.R.; SQUIRE, C.R. Effects of atmosphere saturation deficit on the stomatal conductance on pearl millet (Pennisetum typhoideum S. e H.) and groundnut (Arachis hypogaea L.). Journal of Experimental Botany, Oxford, v.30, p.935-945, 1979.

BUCKMAN, H.O.; BRADY, N.C. Natureza e propriedades dos solos. 3.ed. Rio de Janeiro : Freitas Bastos, 1974. $549 \mathrm{p}$

ECKSTEIN, K.; ROBINSON, J.C. Physiological responses of banana (Musa AAA, Cavendish 
subgroup) in the subtropics. VI. Seasonal responses of leaf gas exchange to short-term water stress. Journal of Horticultural Science, Kent, v.71, n.5, p.679-692, 1996.

FARQUHAR, G.D.; SHARKEY, T.D. Stomatal conductance and photosynthesis. Annual Review of Plant Physiology, Palo Alto, v.33, p.317-345, 1982.

FUNDAÇÃO DE DESENVOLVIMENTO MUNICIPAL DO INTERIOR DE PERNAMBUCO (Recife, PE). Histórico do município de Paudalho. In:__ Perfil municipal do interior de Pernambuco. Recife, 1994. p.639-642.

GALYUON, I.K.A.; McDAVID, C.R.; LOPEZ, F.B.; SPENCE, J.A. The effect of irradiance level on cocoa (Theobroma cacao L.). I. Growth and leaf adaptations. Tropical Agriculture, St. Augustine, v.73, n.1, p.23-28, 1996a

GALYUON, I.K.A.; McDAVID, C.R.; LOPEZ, F.B.; SPENCE, J.A. The effect of irradiance level on cocoa (Theobroma cacao L.). II. Gas exchange and chlorophyll fluorescence. Tropical Agriculture, St Augustine, v.73, n.1, p.29-33, 1996 b.

HSIAO, T.C. Plant responses to water stress. Annual Review of Plant Physiology, Palo Alto, v.24, p.519570,1973

KANNO, S.S. Curso diário e sazonal das trocas gasosas e potencial hídrico foliar em três espécies lenhosas do cerrado: Anacardium nanum St. Hill, Andira humilis Marth. ex Benth e Eriotheca gracilipes (K. Schum) A. Robins. São Carlos : Universidade Federal de São Carlos, 1993. 100p. Dissertação de Mestrado.

KÖRNER, C.; JUDITH, A.; SCHEEL, A.; BAUER, H. Maximum leaf diffusive conductance in vascular plants. Photosynthetica, Dordrecht, v.13, n.1, p.4582,1979 .

KRIEDEMANN, P.E. Photosynthesis and transpiration as a function of gaseus diffusive resistances in orange leaves. Physiologia Plantarum, Copenhagen, v.24, p.218-225, 1971.

KRIEDEMANN, P.E. Some photosynthetic characteristics of citrus leaves. Australian Journal of Biological Sciences, Collingwood, v.21, p.895905,1968
LARCHER, W. Physiological plant ecology. Berlin : Springer, 1975. 303p.

MACHADO, E.C.; QUAGGIO, J.A.; LAGÔA, A.M.M.A.; TICELLI, M.; FURLANI, P.R. Trocas gasosas e relações hídricas em laranjeiras com clorose variegada dos citros. Revista Brasileira de Fisiologia Vegetal, Brasília, v.6, n.1, p.53-57, 1994.

MIELE, A. Influência do sistema de condução na evolução dos açúcares redutores e da acidez total durante a maturação da uva: relação com área foliar, radiação solar e fotossíntese. Revista Brasileira de Fisiologia Vegetal, Brasília, v.1, n.1, p.32-40, 1989.

MILLAR, A.A.; GARDNER, W.R.; GOLTZ, S.M. Internal water status and water transport in seed onion plants. Agronomy Journal, Madison, v.63, n.2, p.779-784, 1971

NOGUEIRA, R.J.M.C.; MORAES, J.A.P.V. Ecofisiologia da acerola. I. Transpiração, resistência difusiva e temperatura foliar. In: SEMINÁRIO REGIONAL DE ECOLOGIA, 8., 1996, São Carlos. Anais. São Carlos : Universidade de São Carlos-Programa de Ecologia e Recursos Naturais, 1997. v.3, cap.1, p.1085-1091.

PEREIRA NETTO, A.B.; HAY, J.D. Fotossíntese em Caryocar brasiliense no cerrado. Revista Brasileira de Botânica, São Paulo, v.9, p.259-262, 1986

PEREZ, S.C.J.G. de A. Curso diário e sazonal do potencial da água e da condutância difusiva ao vapor em plantas lenhosas de um cerradão. São Carlos : Universidade Federal de São Carlos, 1984. 344p. Dissertação de Mestrado.

PEREZ, S.C.J.G.A.; MORAES, J.A.P.V. Determinações de potencial hídrico, condutância estomática e potencial osmótico em espécies dos estratos arbóreo, arbustivo e herbáceo de um cerradão. Revista Brasileira de Fisiologia Vegetal, Brasília, v.3, n.1, p.2737, 1991

PRADO, C.H.B. de A. Capacidade fotossintética de algumas espécies lenhosas do cerrado sob condições de campo. São Carlos : Universidade de São Carlos, 1994. 131p. Tese de Doutorado.

RIEGER, M. Growth, gas exchange, water uptake and drought response of seedling and cutting propagated peach and citrus rootstocks. American Society for Horticultural Science Journal, Alexandria, v.117, n.2, p.834-840, 1992 
ROCHA, A.M. dos S.; MORAES, J.A.P.V. Influência do estresse hídrico sobre as trocas gasosas em plantas jovens envasadas de Stryphnodendron adstringens (Mart.) Coville. Revista Brasileira de Fisiologia Vegetal, Brasília, v.9, n.1, p.43-48, 1997.

SALINAS, A.R.; ZELENER, N.; CRAVIOTTO, R.M.; $\mathrm{BISARO}, \mathrm{V}$. Respuestas fisiológicas que caracterizan el comportamiento de diferentes cultivares de soja a la deficiencia hídrica en el suelo. Pesquisa Agropecuária Brasileira, Brasília, v.31, n.5, p.331338, maio 1996

SCHOLANDER, P.F; HAMMEL, H.T.; HEMINGSEN, E.A.; BRADSTREET, E.D. Hydrostatic pressure and osmotic potentials in leaves of mangroves and some other plants. National Academy of Sciences of the United States of America Proceedings, Washington, v.51, p.119-125, 1965.

SCHULZE, E.D.; LANGE, O.L.; BUSCHBOM, H.; KAPPEN, L.; EVENARI, M. Stomatal responses to changes in humidity in plants growing in the desert. Planta, Berlin, v.108, p.259-270, 1972.

VIANELLO, R.L.; ALVES, A.R. Meteorologia básica e aplicações. Viçosa : Imprensa Universitária, 1991. $449 \mathrm{p}$.

VU, J.C.V.; YELENOSKY, G. Water deficit and associated changes in some photosynthetic parameters in leaves of 'Valencia' orange (Citrus sinensis L. Osbeck) Plant Physiology, Rockville, v.88, p.375-378, 1988 